\title{
Many-body localization phase in a spin-driven chiral multiferroic chain
}

\author{
S. Stagraczyński, ${ }^{1}$ L. Chotorlishvili, ${ }^{1}$ M. Schüler, ${ }^{1,2}$ M. Mierzejewski, ${ }^{3}$ and J. Berakdar ${ }^{1}$ \\ ${ }^{1}$ Institut für Physik, Martin-Luther-Universität Halle-Wittenberg, 06099 Halle, Germany \\ ${ }^{2}$ Department of Physics, University of Fribourg, 1700 Fribourg, Switzerland \\ ${ }^{3}$ Institute of Physics, University of Silesia, 40-007 Katowice, Poland \\ (Received 6 April 2017; revised manuscript received 8 August 2017; published 25 August 2017)
}

\begin{abstract}
Many-body localization (MBL) is an emergent phase in correlated quantum systems with promising applications, particularly in quantum information. Here, we unveil the existence and analyze this phase in a chiral multiferroic model system. Conventionally, MBL occurrence is traced via level statistics by implementing a standard finite-size scaling procedure. Here, we present an approach based on the full distribution of the ratio of adjacent energy spacings. We find a strong broadening of the histograms of counts of these level spacings directly at the transition point from MBL to the ergodic phase. The broadening signals reliably the transition point without relying on an averaging procedure. The fast convergence of the histograms even for relatively small systems allows monitoring the MBL dynamics with much less computational effort. Numerical results are presented for a chiral spin chain with a dynamical DzyaloshinskiiMoriya interaction, an established model to describe the spin excitations in a single-phase spin-driven multiferroic system. The multiferroic MBL phase is uncovered and it is shown how to steer it via electric fields.
\end{abstract}

DOI: 10.1103/PhysRevB.96.054440

\section{INTRODUCTION}

Disorder may localize propagating waves. This phenomenon, first unraveled for noninteracting electronic systems [1], applies also in a general setting involving even classical waves [2-4]. For the ground state of a correlated system disorder is also of a great importance and may inhibit conductance [5]. Between these two regimes interesting phenomena emerge as well: For a certain disorder strength, a correlated excited system may form a MBL phase [6-12] with distinct properties such as boundary-law entropy, localization protected order, and vanishing DC (even non-linear) conductivity with MBL mobility edge between the localized and the thermalized (we call it also ergodic) phase. There are indications of the quantum nature of MBL [13]. Experiments on ultracold atoms in optical lattices [14-16] and trapped ions [17] provided evidence of MBL. Here, we propose studying the signature of MBL in electromagnon excitations of spin-driven multiferroic oxides [18]. A possible MBL is expected to hinder the electromagnon transport, which can be traced down, for instance, as in Ref. [19]. One currently discussed question is how the spectrum of a system with MBL phase is distributed. For integrable systems this issue has long been in the focus of research with many interesting findings (cf., for instance, Refs. [20-27]). Generically, integrable systems with a large number of degrees of freedom turn chaotic for a weak perturbation. For interacting systems, the MBL phase is possibly mappable onto a model with fewer degrees of freedom [28,29] and hence is resistent to perturbations until reaching a critical perturbation strength destroying the MBL phase. This scenario can be assessed by indicators that signal the transition from the MBL to the thermalized (ergodic) phase. Conventionally, the levels statistics behavior is used for this purpose [30]. In the ergodic phase the level spacing follows a Wigner distribution function, while in the MBL phase it obeys a Poisson distribution. A key quantity is the disorder average of the ratio

$$
\begin{aligned}
r_{n} & =\min \left(\delta_{n}, \delta_{n-1}\right) / \max \left(\delta_{n}, \delta_{n-1}\right), \\
r & =\frac{1}{N-2} \sum_{n=3}^{N} r_{n},
\end{aligned}
$$

where $\delta_{n}=E_{n}-E_{n-1}$ is the distance between two neighbor energy levels labelled by $n$ and $N$ is the number of eigenstates. In the ergodic phase for the Gaussian orthogonal ensemble (GOE) $r_{\mathrm{GOE}}=0.5307$ is found [31], while in the $\mathrm{MBL}$ phase $r_{\text {Poisson }}=0.3863$. The disorder strength has a strong influence on the system's spectral characteristics. Typically, the transition to the MBL phase sets in at a certain critical strength of disorder as deduced from conventional finite-size scaling analysis, assumed to apply also to the MBL case $[8,10]$. The spectrum is obtained via exact diagonalization. In this work, we present a new method based on the histograms of counts (hereafter, histograms) of the interlevel distances. The transition point is marked by a broadening of the histograms and enhanced fluctuations. We will be dealing with a system with a mixed symmetry having the Hamiltonian (GUE denotes Gaussian unitary ensemble)

$$
\hat{H}_{\mathrm{total}}=\hat{H}_{\mathrm{GOE}}+\lambda \hat{H}_{\mathrm{GUE}}
$$

Systems with mixed GOE/GUE symmetries are of high interest and widely studied in the recent literature [32]. Depending on the value of the parameter $\lambda$ the spectrum of the Hamiltonian Eq. (2) displays different features: In the limit of small $\lambda$ the level statistics obey GOE, for large $\lambda$ GUE prevails. Interestingly, in the crossover regime the system shows qualitatively different properties than $\hat{H}_{\mathrm{GOE}}$ and $\hat{H}_{\mathrm{GUE}}$. Particularly for such cases, further methods to explore the MBL phase in systems with mixed complex symmetries are useful and needed. We observed that in spite of the difference of the GOE and GUE statistics, the enhanced fluctuations at 
the MBL transition point bear a universal physical character common for both GOE and GUE symmetries. Thus, our method serves as a useful tool to explore MBL in systems with complex symmetry properties.

The manuscript is structured as follows: In Sec. II, we introduce the helical spin-1/2 Heisenberg model, in the Sec. III, we explore the integrals and the transformation properties of the helical spin-1/2 Heisenberg model. Section IV is dedicated to the MBL phase in the helical spin- $1 / 2$ Heisenberg model, and in Sec. V, we introduce the histograms of counts as a tool for sensing the MBL phase and explore the enhanced fluctuations near the transition point.

\section{HELICAL SPIN-1/2 HEISENBERG MODEL}

MBL phenomena were analyzed for disordered quantum spin chains (cf., for instance, Refs. [8-12]). Our focus here is on a particular system, namely on low-energy excitations of oxide-based, spin-driven multiferroics that were realized experimentally in a chain form $[18,33,34]$ (such as $\mathrm{LiCu}_{2} \mathrm{O}_{2}$ ), and are charge insulators. Such systems exhibit at low temperatures a helical spin order coupled to an effective electric polarization. The ferroelectric polarization order is described by Ref. [18], $\mathbf{P}=g_{\mathrm{ME}} \sum_{i=1}^{L}\left\langle\mathbf{e}_{x} \times\left(\hat{\mathbf{S}}_{i} \times \hat{\mathbf{S}}_{i+1}\right)\right\rangle$. Here, $g_{\mathrm{ME}}$ is the strength of the magnetoelectric coupling. The $L$ effective spin-1/2 moments localized at sites $i$ are described by the operators $\hat{\mathbf{S}}_{i}$ and $\mathbf{e}_{x}$ is the spatial unit vector along the $x$ axis connecting $\hat{\mathbf{S}}_{i}$ and $\hat{\mathbf{S}}_{i+1}$. Thus, the low-energy excitations are electromagnons. A possible $\mathrm{MBL}$ phase has a multiferroic nature and is controllable by a magnetic B field (we assumed it applied along the $z$ axis) or an electric field $\mathbf{E}$ (applied below along the $y$ axis) that couples to $\mathbf{P}$. Such a scenario is well captured by the low-energy, effective spin-1/2 Hamiltonian with a dynamical DM interaction [18,35]:

$$
\begin{aligned}
\hat{H}= & J_{1} \sum_{i=1}^{L} \hat{\mathbf{S}}_{i} \cdot \hat{\mathbf{S}}_{i+1}+J_{2} \sum_{i=1}^{L} \hat{\mathbf{S}}_{i} \cdot \hat{\mathbf{S}}_{i+2} \\
& +\sum_{i=1}^{L} B_{i}^{z} \hat{S}_{i}^{z}+D \sum_{i=1}^{L}\left(\hat{\mathbf{S}}_{i} \times \hat{\mathbf{S}}_{i+1}\right)_{z}, D=E_{y} g_{\mathrm{ME}}
\end{aligned}
$$

Note, $D$ combines the effect of the electric field and the magnetoelectric coupling. The nearest-neighbor exchange interaction is ferromagnetic $J_{1}<0$, while the next-nearestneighbor one is antiferromagnetic $J_{2}>0$ leading in general to a frustrated spin order. For $D=0$ and uniform magnetic field $B_{i}^{z}=B^{z}, \hat{H}$ is of the Majumdar-Ghosh type [36]. Breaking the $\mathrm{SU}(2)$ symmetry, for instance, by an intrinsic anisotropy, the expectation value of the vector chirality $\boldsymbol{\kappa}_{i}=\left\langle\hat{\mathbf{S}}_{i} \times \hat{\mathbf{S}}_{i+1}\right\rangle$ turns finite (see, e.g., Refs. [37,38]), signaling the emergence of a spin ordering in the $x y$ projection. The dependence on $D$ implies a variation in $E_{y}$ for the material-specific $g_{\mathrm{ME}}$. For $J_{2}=0$, the anisotropic Heisenberg chain is retrieved. We note that electromagnon excitations may soften the phonon modes due to different possible types of magnetoeleastic couplings such as exchange-striction $[39,40]$. This effect in relation to MBL is not addressed here. Generally, coupling MBL systems to an incoherent environment may restore ergodicity washing out MBL signatures [41-44]. Here, we qualitatively assess the role of phonon modes on MBL by disordering $D$, as discussed below. Computationally, we are able to deal with only small chains as compared to experiment, for instance on $\mathrm{LiCu}_{2} \mathrm{O}_{2}$ chain. On the other hand, our model is versatile and captures also the noncollinear spiral order evidenced experimentally in size-selected, individual biatomic Fe chains on the $(5 \times 1)-\operatorname{Ir}(001)$ surface [45].

\section{INTEGRALS OF THE SYSTEM AND TRANSFORMATION PROPERTIES}

Our system $\hat{H}$ has certain integrals of motion. It is straightforward to show that the total spin component $\hat{S}^{z}=$ $\sum_{i=1}^{L} \hat{S}_{i}^{z}$ commutes with $\hat{H}$. Therefore, $\hat{H}$ is block-diagonal. Each block is identified via the conserved total spin component $\hat{S}^{z}$. Of special interest is the largest subspace of states $\left|\Psi_{n}\right\rangle$ obeying $\hat{S}^{z}\left|\Psi_{n}\right\rangle=M\left|\Psi_{n}\right\rangle$ with $M=0$ for even $L$ or $M=1$ for odd $L$, respectively. A uniform magnetic field $B_{i}^{z}=B^{z}$ shifts equally the eigenvalues in each subspace and has no prominent effect on the interlevel distance $r_{n}$, while randomness incorporated in the magnetic field $B_{i}^{z} \in\langle-h, h\rangle$ can induce a qualitative change of the spectral properties from Wigner-Dyson to Poisson level spacing statistics. The strength of disorder is measured on a scale set by $J_{1}$. In what follows we work with dimensionless units such that $J_{1}=1, J_{2} \rightarrow J_{2} / J_{1}$, $D \rightarrow D / J_{1}, B \rightarrow B / J_{1}$. For simplicity, we omit the factor of $1 / 2$ in front of spin operator $\hat{\mathbf{S}} \equiv \hat{\sigma}$ implying an extra rescaling $J_{1,2}=J_{1,2} / 4, D, B^{z}=D, B^{z} / 2$. Depending on the experimental realization different boundary conditions have to be taken into account. For open boundary conditions, a unitary local rotation of spins $\hat{S}_{k}^{+} \rightarrow \hat{S}_{k}^{+} e^{-i k \Theta}, \hat{S}_{k}^{-} \rightarrow \hat{S}_{k}^{-} e^{i k \Theta}$ around the $z$ axis by the angle $\Theta=-\arctan \left(D / J_{1}\right)$ converts the Hamiltonian Eq. (3) to

$$
\begin{aligned}
\hat{H}_{T}= & J_{1} \sum_{i=1}^{L} \hat{S}_{i}^{z} \hat{S}_{i+1}^{z}+\frac{J_{1}^{\prime}}{2} \sum_{i=1}^{L}\left(\hat{S}_{i}^{+} \hat{S}_{i+1}^{-}+\hat{S}_{i}^{-} \hat{S}_{i+1}^{+}\right) \\
& \times J_{2} \sum_{i=1}^{L} \hat{S}_{i}^{z} \hat{S}_{i+2}^{z}+\frac{J_{2}^{\prime}}{2} \sum_{i=1}^{L}\left(\hat{S}_{i}^{+} \hat{S}_{i+2}^{-}+\hat{S}_{i}^{-} \hat{S}_{i+2}^{+}\right) \\
& -\sum_{i=1}^{L} B_{i}^{z} \hat{S}_{i}^{z}-D^{\prime} \sum_{i=1}^{L}\left(\hat{\mathbf{S}}_{i} \times \hat{\mathbf{S}}_{i+2}\right)_{z} .
\end{aligned}
$$

Here, $\quad J_{1}^{\prime}=\sqrt{J_{1}^{2}+D^{2}}, \quad J_{2}^{\prime}=J_{2}\left(J_{1}^{2}-D^{2}\right) /\left(J_{1}^{2}+D^{2}\right), \quad D^{\prime}=$ $D J_{1} J_{2} /\left(J_{1}^{2}+D^{2}\right)$. As evident, for $J_{2}=0$ the DM interaction term disappears and the Hamiltonian is equivalent to the $X X Z$ model; whereas for twisted periodic boundary conditions, a reminiscent of a DM interaction remains [46].

\section{MANY-BODY LOCALIZATION WITH MIXED SYMMETRIES}

For systems of mixed GOE/GUE symmetries, Eq. (2), three asymptotic cases are of interest: (a) $\hat{H}_{\mathrm{GOE}}$ term is the dominant term and $\hat{H}_{\mathrm{GUE}}$ is the small perturbation, (b) both terms $\hat{H}_{\mathrm{GOE}}$ and $\hat{H}_{\mathrm{GUE}}$ are of equal strength, (c) $\hat{H}_{\mathrm{GUE}}$ term is the dominant term and $\hat{H}_{\mathrm{GOE}}$ is a small perturbation. Cases (a) and (c) are relatively simple and well captured by a standard finite-size scaling procedure. For case (b), the level statistics cannot be identified in terms of GOE and GUE. Systems of different size $L$ manifest nonequivalence and different features. The role 


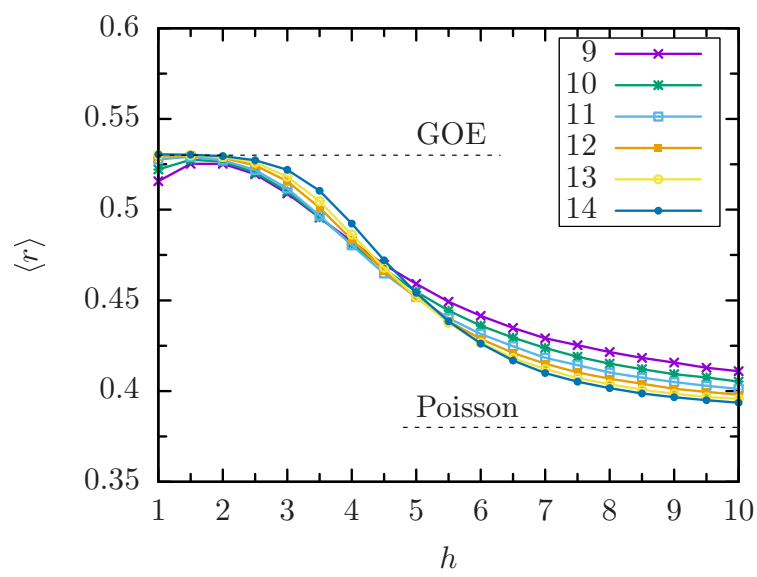

FIG. 1. Level statistics for a ferromagnetic nearest neighbor interaction $J_{1}=-1$, as a function of the strength of disorder $h$ of the magnetic field $B^{z} \in\langle-h, h\rangle$. The DM interaction term is zero and the system exhibits a precise GOE symmetry.

of GUE enhances at larger $L$ (see below). In the numerically inaccessible limit $L \rightarrow \infty$, the system is characterized by GUE statistics. However, the collapse of different data for different numerically accessible finite $L$ to a single universal curve (as expected by a finite-size scaling procedure) is generally not achievable. Here we focus on periodic boundary conditions. Prior implementing a finite-size scaling procedure we present results for systems of different lengths $L=9,10, . .14$.

Figure 1 shows the statistically averaged nearest-neighbor interlevel distances for different strengths of disorder $h$ of the magnetic field $B^{z} \in\langle-h, h\rangle$. In particular, Fig. 1 corresponds to case (a) when the symmetry of the system is precisely identified as GOE and the DM term is zero. As inferred for zero field, the statistics obtained for systems of different lengths perfectly fits with a standard GOE $\langle r\rangle=0.53$. Increasing the strength of disorder $h$, the system performs a transition to the Poisson statistics and this transition grasps the concept of MBL

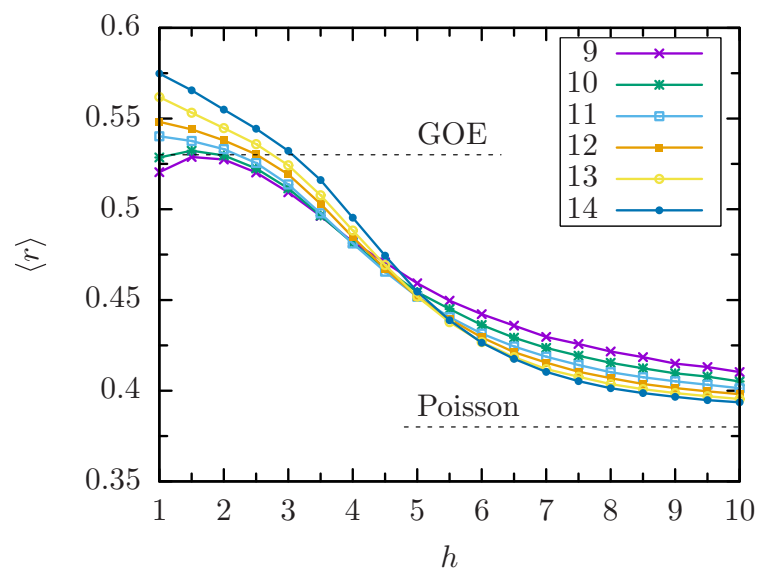

FIG. 2. Level statistics for the ferromagnetic nearest neighbor interaction $J_{1}=-1$, as a function of the strength of disorder $h$ of the magnetic field $B^{z} \in\langle-h, h\rangle$. The DM interaction term is small $D=$ 0.01 . The dominant symmetry of the system is GOE. The influence of GUE enhances with the system's size $L$. phase. Figure 2 shows the results for the case when the DM term is small and GOE is the main symmetry of the system, while GUE is a perturbation. For this case, we conclude that the larger the system size $(L=14)$ the more important is the contribution of the DM term. Note that a finite-size scaling procedure relies on the collapse of different data for different $L$ to a single universal curve. Here we face a problem because for large $L$ the deviation from GOE is more prominent. For stronger DM term the deviations from GOE increase (see Fig. 3). For $D=0.05$, the system turns into mainly GUE type and GOE is a perturbation; see Fig. 4. However, this case still corresponds to case (b), meaning a mixed complex symmetry and the finite-size scaling procedure fails. Only for unrealistically large $D=0.2$ we have the case (c) (the $\hat{H}_{\mathrm{GUE}}$ term is dominant and $\hat{H}_{\mathrm{GOE}}$ is a small perturbation). For a strong DM interaction $D=0.2$ one observes a perfect switching from the GOE to the GUE level statistics and a finite-size scaling procedure provides reasonable results [cf. Figs. 5(a) and 5(c)]. Thus, finite-size scaling is reliable when the DM interaction term is either zero or large enough and the symmetry of the system is mainly GOE or GUE, respectively.

Each curve corresponds to a system with a different size and is obtained by means of an extensive averaging procedure over the ensemble of realizations of random disorder (up to 10.000 realizations per single point of the curve). For a detailed analysis of the dependence on the critical strength of randomness $h_{c}$, we employ the scaling function [8] $g\left[L^{1 / v}\left(h-h_{c}\right)\right]$, which allows collapsing all data to a single curve. Thus, for a given exponent $v$ we identify a critical disorder strength $h_{c}$. Figure 5(b) indicates a critical disorder strength of $h_{c}=4.7$ in the case of a ferromagnetic nearest-neighbor interaction. The next-nearest-neighbor antiferromagnetic spin interaction causes frustration and opens an extra channel for the energy redistribution in the system enhancing the critical disorder required for the MBL phase to $h_{c}=6.2$. For ferromagnetic $J_{1} \neq 0$ and DM interaction $D \neq 0$, and only $J_{2}=0$, the required disorder strength for MBL phase is noticeably decreased $h_{c}=4.4$. It was proposed [47] that the transition to the MBL phase in the $X X Z$ model occurs for $h>J_{1}$ with numerical results [48] indicating $h>3.5 J_{1}$. Implementing the scaling procedure, we infer $h_{c}=4.6 J_{1}$.

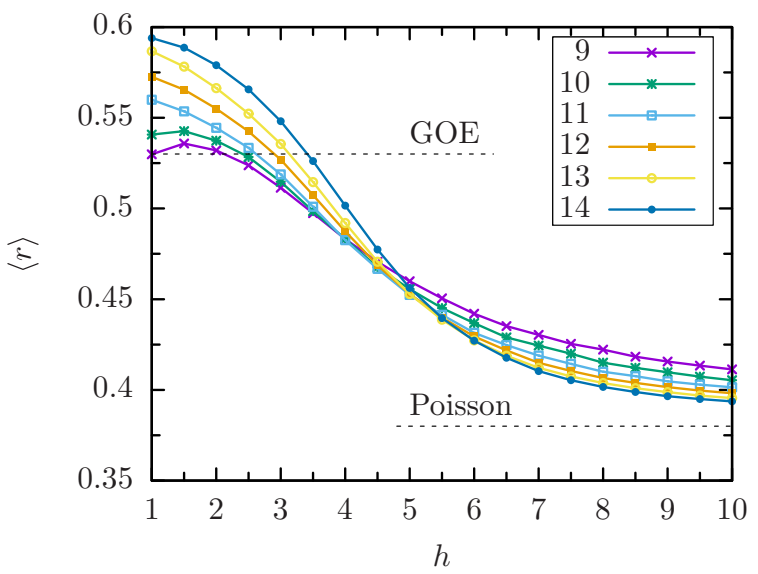

FIG. 3. The same as for Fig. 2 but for $D=0.02$. 


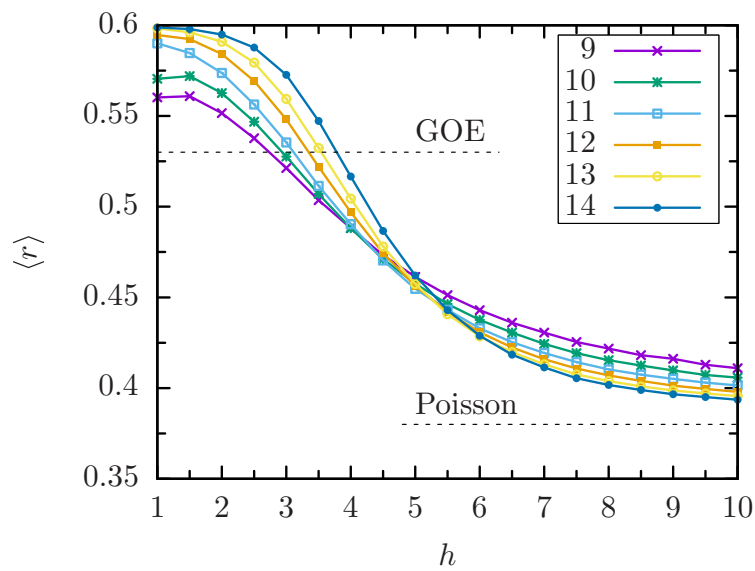

FIG. 4. Level statistics for $J_{1}=-1$, as a function of the strength of disorder $h$ of the magnetic field $B^{z} \in\langle-h, h\rangle$. The DM interaction term is stronger $D=0.05$. The symmetry of the system is mixed.

As follows from Eq. (4) for $J_{2}=0$, the $X X Z$ model is retrieved with an effective $X X$ constant $J_{1}^{\prime}=\left(\sqrt{J_{1}^{2}+D^{2}}\right) / 2$. For $D<J_{1}$ and $J_{1}^{\prime}<J_{1}$, the $X X Z$ system is gapped in line with the observation that MBL is reached at weaker disorder. For $J_{2} \neq 0$ the equivalence with the $X X Z$ model no longer holds and the DMI interaction extends the ergodic phase to $h_{c}=7.3$.

As Fig. 5 shows, the intrinsically complex nature of the Hamiltonian in the presence of a relatively strong DM

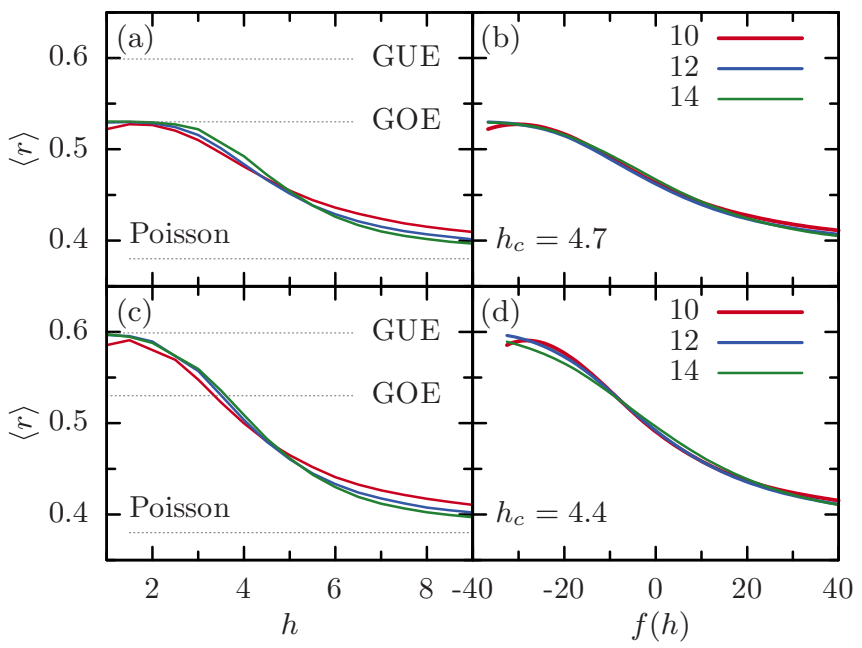

FIG. 5. Level statistics for ferromagnetic nearest neighbor interaction $J_{1}=-1$. For (a) $D=0$. Increasing the randomness range $2 h$ of the magnetic field $B^{z} \in\langle-h, h\rangle$ the system spectrum undergoes a transition from GOE to Poisson distribution, indicating a transition from ergodic to MBL phase. Curves correspond to different system sizes and are obtained by an ensemble average for random disorder. (b) Scaling collapse in the vicinity of the MBL transition is achieved by the scaling function $f(h)=L^{1 / v}\left(h-h_{c}\right), v=1$, $h_{c}=4.7$. (c) With DM interaction $(D=0.2)$. Periodic boundary conditions are implemented and the system is of GUE type. Increasing randomness the system undergoes a transition from GUE to the Poisson distribution. (d) The rescaled phase transition curves from the ergodic to the MBL phase for $D=0.2, h_{c}=4.4, v=1, f(h)=$ $L^{1 / v}\left(h-h_{c}\right)$.

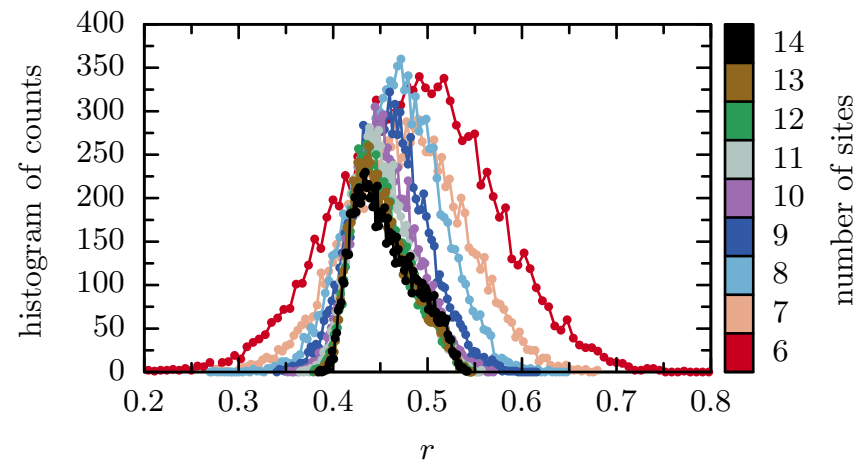

FIG. 6. Histograms of counts for a fixed strength of disorder $h=5$ without DM interaction as a function of the consecutive level spacing $r$. Convergence is indicated for $L=14$.

interaction causes a transition from a GOE to a GUE. As clarified by the mapping to Eq. (4), the complex part of the Hamiltonian is only due to the twisted boundary conditions. Hence, a gradual crossover from the GUE to the GOE with increasing $L$ is expected and confirmed by Fig. 5(c). For this reason, a finite-size scaling is applicable for a large enough disorder.

\section{QUANTUM FLUCTUATIONS AND HISTOGRAMS OF COUNTS}

To formulate a possibly general criterion for MBL that is applicable in such cases, we analyzed the full statistics for each realization $\alpha$ of the random magnetic fields $r^{(\alpha)}$. The histograms corresponding to a counting classification of $r^{(\alpha)}$ for a given disorder strength $h$ is presented on Fig. 7. As can be inferred, the histograms are narrow far away from the MBL transition, while the histograms become particularly broad close to the transition point mimicking the behavior of fluctuations near conventional phase transitions. The histograms become more and more pronounced for increasing

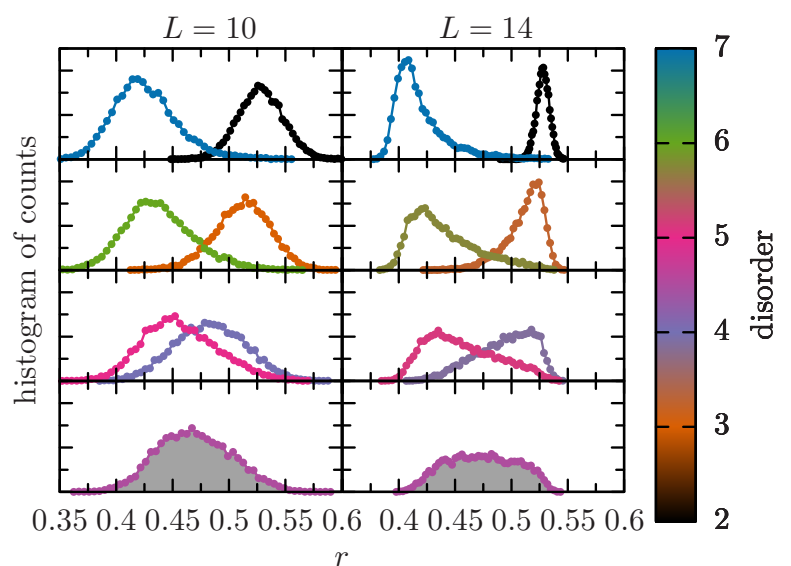

FIG. 7. Histograms of counts for the different strength of disorder $h$ with $J_{1}=-1$ without DM interaction as a function of the consecutive level spacing $r$. Broadening of the histogram corresponds to the critical strength of disorder and to the transition point. The larger $L$ is, the more distinguished the peaks are. 


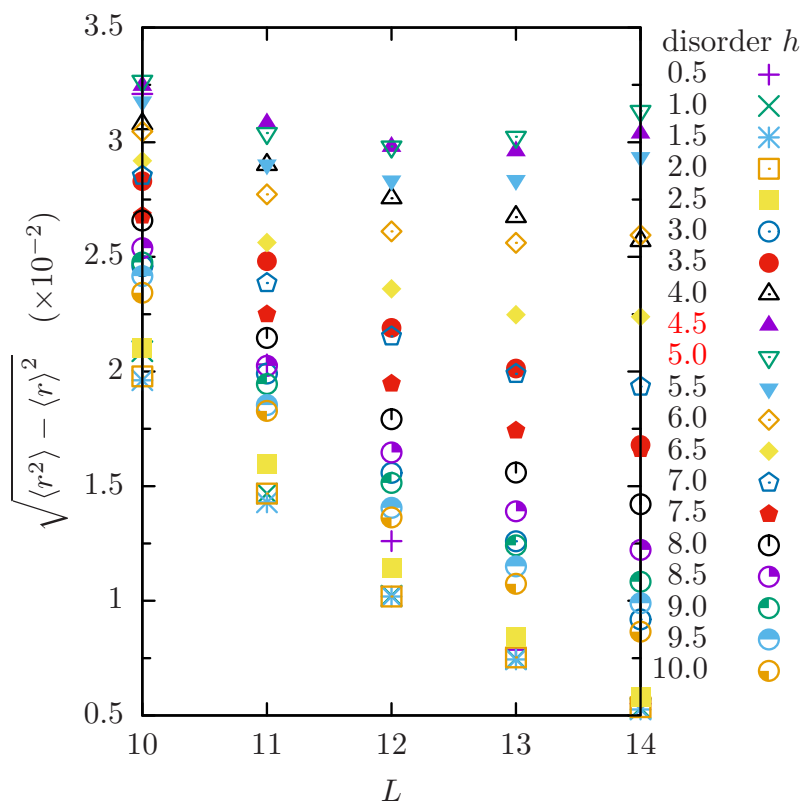

FIG. 8. The fluctuation dependency on system size without $D$ and $J_{1}=-1$. The two datasets marked by red are the nearest to critical disorder.

$L$. Figure 6 demonstrates the convergence of the histograms of counts for the chains of different lengths. As we see already for $L=14$ counts histograms amalgamate underlying that an analysis of the histograms can serve as a further indicator in addition to finite-size scaling. The convergence of histograms even for relatively small systems endorses our method as less computationally demanding which is a major advantageous for exact diagonalization approaches that are considered as well suited for MBL studies. As for the histograms of consecutive level spacing, Fig. 7 illustrates the broadening of the histograms when approaching the transition point between the ergodic and the MBL phases. As evident, the effect of broadening is even more prominent for systems with a larger size Fig. 7. We note that the observed phenomena is not related to a particular type of level statistics but it is rather akin to the transition regime. Away from the transition point

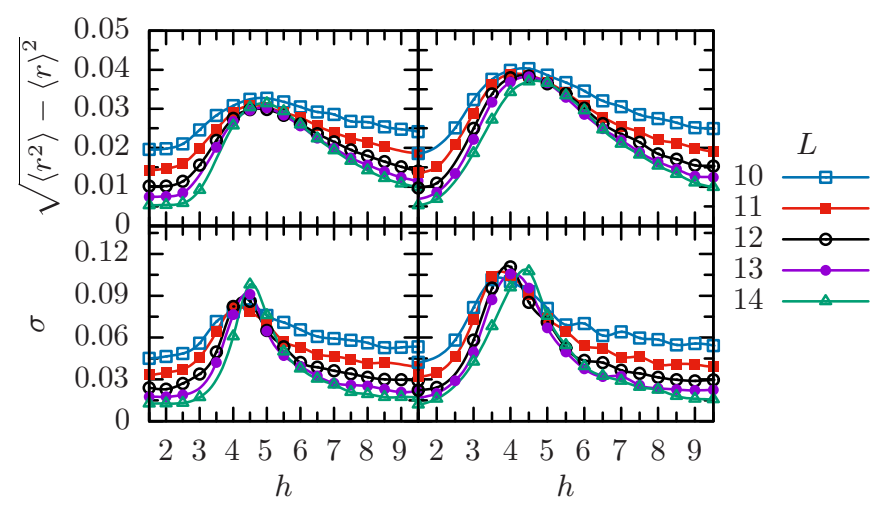

FIG. 9. Full width at half maximum $\sigma$ for the histograms as a function of disorder $h$. The graphs on the right side are with finite DM interaction, $D=0.2$.

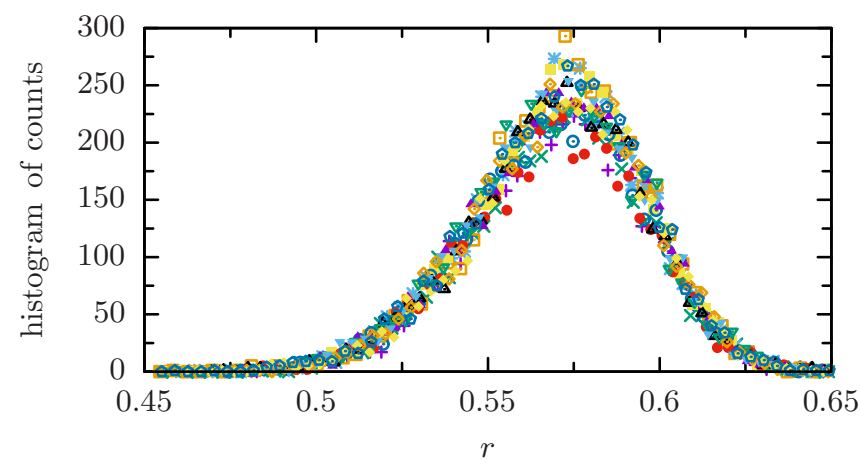

FIG. 10. $J_{1}=-1, D=0.2$, for fixed $h=5$. Additional sitedependent disorder of $10 \%$ in $D$ was taken while different colors and marks refer to various realizations.

on the ergodic side (GOE statistics) and on the MBL phase side (Poisson statistics) the width of histograms are narrower. The broadening is linked to the enhanced quantum fluctuations Fig. 8. This behavior is of a general character and is maintained even after adding next nearest neighbor interaction and DM interaction terms.

Physically, the broadening of histograms is attributable to the enhanced fluctuations near phase transitions (cf. Figs. 8 and 9). Hence, such broadening serves as a further indicator for approaching the MBL phase.

Disorder in the exchange coupling or in $D$ may also occur. The latter [cf. Eq. (3)] can be viewed as random change in $\mathbf{E}$ or a random elastic energy change $\left(\mathbf{E} \cdot \mathbf{P}=g_{\mathrm{ME}} \mathbf{E} \sum_{i=1}^{L}\left\langle\mathbf{e}_{x} \times\right.\right.$ $\left.\left.\left(\hat{\mathbf{S}}_{i} \times \hat{\mathbf{S}}_{i+1}\right)\right\rangle\right)$, and thus, it is important for spin-phononcoupled systems at finite temperatures. Calculations evidence the robustness of the MBL phase against randomizing $D$ within a physically reasonable range, an example is depicted in Fig. 10.

The results obtained for the different values of parameters are listed in the Table I. Note, for several particular values of the DM term that correspond to the mixed GOE/GUE statistics, the MBL phase can be identified through the present method, while a finite-size scaling procedure fails.

TABLE I. The estimated critical disorders $h$ with $J_{1}=-1$, (a) $h_{c}$ from a scaling procedure for $\langle r\rangle$, (b) $h_{\sigma}$ from an analysis of the fullwidth at half-height of the histograms, (c) $h_{f}$ from the analysis of the the fluctuations $\sqrt{\left\langle r^{2}\right\rangle-\langle r\rangle^{2}}$. The left dataset with $L=\{10,12,14\}$ and 4096 realizations for each mean of the consecutive level spacings $\langle r\rangle$. The right dataset was prepared with $L=\{10,11,12,13,14\}$ and 10240 realizations.

\begin{tabular}{lccccccccc}
\hline \hline$J_{2}$ & $D$ & $h_{c}$ & $h_{\sigma}$ & $h_{f}$ & $J_{2}$ & $D$ & $h_{c}$ & $h_{\sigma}$ & $h_{f}$ \\
\hline & & 4.6 & 4.5 & 4.8 & & & 4.7 & 4.5 & 4.8 \\
$\frac{1}{4}$ & & 6.2 & 5.3 & 5.7 & & $\frac{1}{100}$ & 5.0 & 4.5 & 4.8 \\
$-\frac{1}{4}$ & & 5.4 & 4.6 & 5.2 & & $\frac{1}{50}$ & 5.3 & 4.4 & 4.6 \\
& $\frac{1}{5}$ & 4.4 & 4.4 & 4.7 & & $\frac{1}{20}$ & 5.6 & 4.0 & 4.5 \\
$\frac{1}{4}$ & $\frac{1}{5}$ & 7.3 & 5.0 & 5.5 & & $\frac{1}{10}$ & 5.4 & 4.2 & 4.6 \\
$-\frac{1}{4}$ & $\frac{1}{5}$ & 6.4 & 4.9 & 5.1 & & $\frac{1}{5}$ & 4.3 & 4.4 & 4.7 \\
\hline \hline
\end{tabular}




\section{CONCLUSIONS}

Summarizing, our numerics and analysis evidence MBL phase in a chiral multiferroic chain. A new, general indicator for approaching the MBL phase is identified and tested against conventional procedures. In view of the experimental feasibility of materials and settings, the predictions might have some signatures experimentally, for instance, through investigating the transport and excitation spectrum of electromagnons.

\section{ACKNOWLEDGMENTS}

We thank Markus Heyl for fruitful discussions and numerous comments on the manuscript. We acknowledge financial support from the Deutsche Forschungsgemeinschaft through SFB 762, and BE 2161/5-1. M.M. acknowledges support by the National Science Centre, Poland via Project No. 2016/23/B/ST3/00647.

\section{APPENDIX: SCALING PROCEDURE}

In Ref. [8] a systematic analysis of the fitting windows and system sizes in relation to the critical disorder $h_{c}$ and $v$ was performed. For the histograms windowing of the energy spectrum from $S^{z}=0$ block causes equalizing disproportions of the histograms from different phases. Therefore, the full spectrum of the biggest block is considered. Although, for a bigger system size the middle part of the spectrum can be successfully used. The level statistics of $\langle r\rangle$ as a function of disorder $h$ are scaled with $f_{L}(h)=L^{1 / v}\left(h-h_{c}\right)$, where $v=1$ was assumed. For best fitting parameter $h_{c}$, the scaling procedure can be supported by a minimizing function $w(h)$,

$$
w=\sum_{L, L^{\prime}} \int_{h_{1}}^{h_{2}}\left|\langle r\rangle\left(f_{L}(h)\right)-\langle r\rangle\left(f_{L^{\prime}}(h)\right)\right| d h,
$$

where $h_{1}$ and $h_{2}$ are defined by the common integration domain $h_{1}=\max \left(L_{i}^{\frac{1}{v}}\left(a-h_{c}\right)\right), h_{2}=\min \left(L_{i}^{\frac{1}{v}}\left(b-h_{c}\right)\right)$, where $L_{i}$ denote the system sizes to be analyzed and $a=1, b=10$ are boundaries limit for unscaled data set.

The critical disorder also can be inferred by minimizing the distances of the peaks positions for $\langle r\rangle$ first derivatives, which is more accurate than minimizing global overlaps, especially with nonzero DM interaction. With Eq. (A1), a proper adjusting of integration limits is required.
[1] P. W. Anderson, Phys. Rev. 109, 1492 (1958).

[2] M. Segev, Y. Silberberg, and D. N. Christodoulides, Nat. Photonics 7, 197 (2013).

[3] H. Hu, A. Strybulevych, J. Page, S. Skipetrov, and B. Van Tiggelen, Nat. Phys. 4, 945 (2008).

[4] A. Lagendijk, B. van Tiggelen, and D. S. Wiersma, Phys. Today 62, 24 (2009).

[5] Electron-electron Interactions in Disordered Systems, edited by M. Pollak and A. L. Efros (North-Holland, Amsterdam, 1984).

[6] D. M. Basko, I. L. Aleiner, and B. L. Altshuler, Ann. Phys. 321, 1126 (2006).

[7] L. Zhang, B. Zhao, T. Devakul, and D. A. Huse, Phys. Rev. B 93, 224201 (2016).

[8] D. J. Luitz, N. Laflorencie, and F. Alet, Phys. Rev. B 91, 081103(R) (2015); 93, 060201(R) (2016).

[9] R. Vasseur, A. J. Friedman, S. A. Parameswaran, and A. C. Potter, Phys. Rev. B 93, 134207 (2016).

[10] J. A. Kjäll, J. H. Bardarson, and F. Pollmann, Phys. Rev. Lett. 113, 107204 (2014).

[11] M. Serbyn and J. E. Moore, Phys. Rev. B 93, 041424(R) (2016).

[12] For an overview we refer to the topical issue Many-Body Localization, edited by J. H. Bardarson et al. Ann. Phys. (2017).

[13] V. Oganesyan, A. Pal, and D. A. Huse, Phys. Rev. B 80, 115104 (2009).

[14] M. Schreiber et al., Science 349, 842 (2015).

[15] J.-Y. Choi et al., Science 352, 1547 (2016).

[16] S. S. Kondov, W. R. McGehee, W. Xu, and B. DeMarco, Phys. Rev. Lett. 114, 083002 (2015).

[17] J. Smith et al., Nat. Phys. 12, 907 (2016).

[18] H. Katsura, N. Nagaosa, and A. V. Balatsky, Phys. Rev. Lett. 95, 057205 (2005); M. Mostovoy, ibid. 96, 067601 (2006); I. A. Sergienko and E. Dagotto, Phys. Rev. B 73, 094434 (2006); Y. Tokura, S. Seki, and N. Nagaosa, Rep. Prog. Phys. 77, 076501
(2014); Y. Tokura and S. Seki, Adv. Mater. 22, 1554 (2010); K. F. Wang, J. M. Liu, and Z. F. Ren, Adv. Phys. 58, 321 (2009).

[19] H.-B. Chen, Y.-Q. Li, and J. Berakdar, J. Appl. Phys. 117, 043910 (2015)

[20] B. Bertini, F. H. L. Essler, S. Groha, and N. J. Robinson, Phys. Rev. Lett. 115, 180601 (2015).

[21] M. Rigol, V. Dunjko, V. Yurovsky, and M. Olshanii, Phys. Rev. Lett. 98, 050405 (2007).

[22] E. Ilievski, J. De Nardis, B. Wouters, J.-S. Caux, F. H. L. Essler, and T. Prosen, Phys. Rev. Lett. 115, 157201 (2015); G. Misguich, V. Pasquier, and J.-M. Luck, Phys. Rev. B 94, 155110 (2016).

[23] B. Pozsgay, M. Mestyán, M. A. Werner, M. Kormos, G. Zaránd, and G. Takács, Phys. Rev. Lett. 113, 117203 (2014).

[24] M. Mierzejewski, P. Prelovšek, and T. Prosen, Phys. Rev. Lett. 113, 020602 (2014).

[25] J. Eisert, M. Friesdorf, and C. Gogolin, Nat. Phys. 11, 124 (2015).

[26] C. Gogolin and J. Eisert, Rep. Prog. Phys. 79, 056001 (2016).

[27] L. D’Alessio, Y. Kafri, A. Polkovnikov, and M. Rigol, Adv. Phys. 65, 239 (2016).

[28] M. Serbyn, Z. Papic, and D. A. Abanin, Phys. Rev. Lett. 111, 127201 (2013).

[29] D. A. Huse, R. Nandkishore, and V. Oganesyan, Phys. Rev. B 90, 174202 (2014).

[30] B. Bauer and C. Nayak, J. Stat. Mech.: Theory Exp. (2013) P09005; V. Oganesyan and D. A. Huse, Phys. Rev. B 75, 155111 (2007); A. Pal and D. A. Huse, ibid. 82, 174411 (2010); C. R. Laumann, A. Pal, and A. Scardicchio, Phys. Rev. Lett. 113, 200405 (2014).

[31] F. Haake, Quantum Signatures of Chaos (Springer, Berlin, 2000).

[32] S. Schierenberg, F. Bruckmann, and T. Wettig, Phys. Rev. E 85, 061130 (2012); A. Rycerz, Phys. Rev. B 85, 245424 (2012). 
[33] S. Park, Y. J. Choi, C. L. Zhang, and S.-W. Cheong, Phys. Rev. Lett. 98, 057601 (2007).

[34] F. Schrettle, S. Krohns, P. Lunkenheimer, J. Hemberger, N. Büttgen, H.-A. Krug von Nidda, A. V. Prokofiev, and A. Loidl, Phys. Rev. B 77, 144101 (2008).

[35] M. Azimi, L. Chotorlishvili, S. K. Mishra, S. Greschner, T. Vekua, and J. Berakdar, Phys. Rev. B 89, 024424 (2014).

[36] C. K. Majumdar and D. K. Ghosh, J. Math. Phys. 10, 1388 (1969); A. V. Chubukov, Phys. Rev. B 44, 4693 (1991); R. Bursill, G. A. Gehring, D. J. J. Farnell, J. B. Parkinson, T. Xiang, and C. Zeng, J. Phys.: Condens. Matter 7, 8605 (1995).

[37] T. Hikihara, L. Kecke, T. Momoi, and A. Furusaki, Phys. Rev. B 78, 144404 (2008).

[38] J. Sirker, Z. Weihong, O. P. Sushkov, and J. Oitmaa, Phys. Rev. B 73, 184420 (2006).

[39] C. Jia and J. Berakdar, Europhys. Lett. 85, 57004 (2009).

[40] C. Jia and J. Berakdar, Phys. Status Solidi B 247, 662 (2010).

[41] E. Levi, M. Heyl, I. Lesanovsky, and J. P. Garrahan, Phys. Rev. Lett. 116, 237203 (2016).
[42] M. H. Fischer, M. Maksymenko, and E. Altman, Phys. Rev. Lett. 116, 160401 (2016).

[43] M. V. Medvedyeva, T. Prosen, and M. Znidaric, Phys. Rev. B 93, 094205 (2016).

[44] H. P. Lüschen, P. Bordia, S. S. Hodgman, M. Schreiber, S. Sarkar, A. J. Daley, M. H. Fischer, E. Altman, I. Bloch, and U. Schneider, Phys. Rev. X 7, 011034 (2017).

[45] M. Menzel, Y. Mokrousov, R. Wieser, J. E. Bickel, E. Vedmedenko, S. Blügel, S. Heinze, K. von Bergmann, A. Kubetzka, and R. Wiesendanger, Phys. Rev. Lett. 108, 197204 (2012); M. Menzel, Y. Mokrousov, R. Wieser, J. E. Bickel, E. Vedmedenko, S. Blugel, S. Heinze, K. vonBergmann, A. Kubetzka, and R. Wiesendanger, ibid. 112, 047204 (2014); D. Serrate et al., Nat. Nanotechnol. 5, 350 (2010).

[46] M. Bocquet, F. H. L. Essler, A. M. Tsvelik, and A. O. Gogolin, Phys. Rev. B 64, 094425 (2001).

[47] K. Kudo and T. Deguchi, Phys. Rev. B 69, 132404 (2004).

[48] C. L. Bertrand and A. M. Garcia-Garcia, Phys. Rev. B 94, 144201 (2016). 